\title{
Editorial note for the special issue on smart transportation
}

\author{
Bo $\mathrm{Xu}^{1,2} \cdot$ Gautam Thakur ${ }^{2}$
}

Published online: 12 June 2021

(c) The Author(s), under exclusive licence to Springer Science+Business Media, LLC, part of Springer Nature 2021

The Guest Editors would like to present the sixth paper of the special issue, "Mitigating Spoofed GNSS Trajectories through Nature Inspired Algorithm", authored by Saravjeet Singh, Jaiteg Singh, and Sukhjit Singh. This paper proposes an algorithm called NiCS to not only detect GNSS signal spoofing but also counter it. The NiCS algorithm does so by analyzing the difference between a location received from GNSS and a location predicted based on the road network and inertial sensors. Unlike many other existing algorithms that rely on dedicated hardware, NiCS uses inertial sensors, which are widely available in mobile phones nowadays, making the algorithm easy to deploy.

We hope you enjoy reading this paper in addition to the five described in the introduction to the special issue.

Publisher's note Springer Nature remains neutral with regard to jurisdictional claims in published maps and institutional affiliations.

Bo Xu

bo.5.xu@here.com

Gautam Thakur

thakurg@ornl.gov

1 HERE Technologies, Burlington, MA, USA

2 Oak Ridge National Laboratory, Oak Ridge, TN, USA 